\title{
Gridshell structural evaluation criteria based on Upward and Downward Modeling Methods in Karamba3D
}

XXIV International Conference

of the Iberoamerican Society

of Digital Graphics

Medellin | Colombia

\author{
Elisa Bomtempo Matos \\ Universidade Federal de Viçosa | Brasil | elisa.matos@ufv.br
}

Andressa Carmo Pena Martinez

Universidade Federal de Viçosa | Brasil | andressamartinez@gmail.com

\begin{abstract}
Despite the vast number of researches that address Gridshells as regular meshes, there is a lack of studies discussing hybrid meshes. In this context, this paper presents a parametric approach and employs visual algorithms for designing digital gridshells with different mesh patterns. We intend to formulate a methodology for Karamba 3D applications that address the structural performance according to variations in geometric composition, number of props, and construction methods. The work seeks to examine patterns that improve structural performance, through a parallel discussion between Upward and Downward modeling methods. Although the Upward modeling method is the most recurrent in studies on the topic, in this study, the Downward method generated structures with better structural performance.
\end{abstract}

Keywords: Gridshell; Pattern; Geometric Modeling; Structural Design; Structural Optimization.

\section{INTRODUÇÃO}

Gridshells são estruturas reticulares tridimensionais formadas por elementos de barras, compondo uma malha que possibilita a criação de formas complexas, capazes de vencer grandes vãos, com considerável altura (KUNZ; PRAUCHNER, 2015). Elas popularizaram-se nos últimos cinquenta anos, pois são estruturalmente eficientes como coberturas e têm grande potencial para readaptação de espaços existentes (CHILTON; TANG, 2016). Também associam liberdade formal à eficiência estrutural, o que permite vencer grandes distâncias, que atingem cerca de 60 metros, sem a necessidade de apoios intermediários. Adicionalmente, elas permitem uma variedade de materiais construtivos, tais como madeira e aço (CHILTON; TANG, 2016). Para Naicu (2012) e Williams (2014), sua resistência é derivada da forma, geralmente com dupla curvatura, além da relação entre a geometria da malha ou grid, o material e as forças internas atuantes.

O comportamento das cargas diferencia-se de uma shell (casca contínua), para um gridshell (casca formada por uma malha treliçada). Shells transferem cargas em todas as direções, enquanto um gridshell transfere cargas axiais na direção de suas peças, podendo ser necessária a utilização de elementos diagonais que restrinjam o movimento da malha (CARVALHO, 2015). Segundo Toussaint e Andriaenssens (2007; 2014), quando se utilizam elementos diagonais nas peças, consegue-se transferir esforços cortantes, fazendo com que o gridshell trabalhe também de forma semelhante a uma casca contínua.
De modo geral, essas estruturas resistem às cargas por meio de sua forma e composição geométrica das grids, podendo ser cada vez mais complexas, à medida que os software de análise estrutural foram sendo refinados (CHILTON; TANG, 2016).

Essas estruturas podem ser classificadas por sua forma e comportamento em relação às cargas. Forma-ativa é caracterizada principalmente por estruturas que se ajustam ao carregamento, como as tensionadas. Formapassiva, por outro lado, são estruturas que não se ajustam ao carregamento, ou seja, sofrem tração e compressão, mas são estruturas aparentemente rígidas (WILLIAM, 2014).

Esse artigo centra-se, então, no estudo de gridshells com elementos de linhas curvas de forma-passiva. As mais populares nessa categoria são as cúpulas geodésicas, que são modeladas por processo de triangulação, elaboradas por Fuller (DŹWIERZYŃSKA, 2018). No entanto, um desafio que ainda permanece no processo de projeto dessas estruturas é a lacuna entre as formas ainda não estudadas, limitadas pelas propriedades dos materiais e padrões das grids (PONE, 2013). Segundo Rieffel et al. (2009), existem poucos métodos capazes de descobrir as geometrias que são capazes de produzir o caráter autoportante, ou seja, suportam a estrutura de modo independente, sem auxilio de outras complementares.

Sendo assim, este artigo constitui a segunda etapa de uma pesquisa cujo objetivo principal é a elaboração de regras de gramática da forma para a geração de 
gridshells, a partir de estudos de geometrias que favorecem o desempenho estrutural. A pesquisa possui como etapas: (1) revisão de literatura sobre gridshells, gramática da forma, simulação e otimização de desempenho estrutural; (2) desenvolvimento de métodos de simulação de desempenho estrutural em Grasshopper, utilizando os plug-ins Kangaroo e Karamba 3D; (3) análise de composições geométricas das malhas ou grids para o desempenho estrutural de gridshell; (4) elaboração de regras para geração de uma gramática da forma.

Para esta etapa, o artigo apresenta algorítmos visuais (GHX) para a criação de modelos digitais de gridshell com vários padrões de geometrias de malhas. O objetivo é desenvolver uma metodologia aplicada ao Karamba 3D para calcular o desempenho estrutural, segundo as variações de composição geométrica, número de suportes e método de modelagem. Mais especificamente, este trabalho discute o comportamento de gridshells concebidos de modo Ascendente e Descendente, como etapa preliminar para o aprofundamento dos estudos dos padrões de composição geométrica da malha.

\section{PLUG-IN DE ANÁLISE ESTRUTURAL: KARAMBA3D}

O Karamba3D é um plug-in de analise estrutural para o Grasshopper. Ele se baseia na análise de elementos finitos e, por isso, toda a simulação pode ser executada em um mesmo ambiente sem necessidade de transferências entre software. Nele pode-se facilmente atribuir propriedades estruturais aos elementos, pois fornece um menu de seleção simples, além de visualização clara dos resultados por meio de um gráfico de cores na própria estrutura (ELIASSEN; HUSEBY, 2018).

O manual do Karamba3D não explicita a teoria dos elementos de viga (PREISINGER, 2016). Segundo Eliassen e Huseby (2018), Clemens Preisinger, desenvolvedor do Karamba, sugere que seja a teoria de Timoshenko, o que significa que as deformações por cisalhamentos, ou shear deformation, são contabilizadas nas análises. Já para as cascas contínuas, os mesmos autores sugerem que seja a teoria de Kirchhoff, modelo matemático para placas finas em que se busca determinar as tensões e deformações de estruturas sujeitas à forças e momentos (LOVE, 1888). Objetivamente, no Karamba 3D, a casca é dividida por elementos triangulares, cujos nós geram seis conexões. Neste sentido, a resolução da malha, conforme a densidade da triangulação, afeta a precisão dos resultados, assim como o tempo de computação da estrutura (ELIASSEN; HUSEBY, 2018).

\section{MÉTODOS CONSTRUTIVOS DE GRIDSHELLS DE ELEMENTOS EM LINHAS CURVAS}

Nesse estudo, serão analisados os Métodos de Modelagem Ascendente e Descendente para a geração de gridshells. A definição do método é fundamental para o processo de projeto, já que a complexidade da estrutura requer 0 desenvolvimento de peças e encaixes específicos na fase de fabricação.

No processo Ascendente (upward), a malha é montada em uma superfície plana, sendo elevada a partir dos pontos predeterminados, reforçados para gerar a forma final, facilitando a ligação entre os elementos. Em estruturas em que existe a sobreposição de camadas, as malhas são produzidas separadamente e ligadas posteriormente, quando a malha inferior estiver elevada em apoios temporários (CARVALHO, 2015).

Andriaenssens (2014) define esse método construtivo como strained gridshell ou gridshell tensionado, pois durante o içamento da malha, a flexibilidade é crucial para a montagem. No entanto, como o procedimento pode causar flexão das peças, resultando em grandes deslocamentos do gridshell, o autor sugere a adição de elementos na diagonal para garantir a rigidez.

Ao elevar a grelha em pontos específicos, a ação da gravidade faz com que a malha se deforme e produza a curvatura da estrutura proposta. Após a elevação, os apoios são fixados e os nós são reforçados (CARVALHO, 2015). Diferentes técnicas podem ser adotadas para elevar a malha, podendo ser puxada por guindastes ou empurrada por macacos hidráulicos, porém, ambas requerem a verificação adicional da estrutura e exigem maior detalhamento e estudo sobre os pontos que sofrerão o esforço adicional de içamento (PAOLI, 2007).

Segundo Carvalho (2015), durante o processo de construção, os elementos estão mais sujeitos à ruptura, uma vez que erros na seleção dos pontos de apoio podem acarretar tensões estruturais superiores à previsão, levando a quebra das ligações. Essas tensões adicionais podem ser decorrentes do raio de curvatura menor das ripas ou da flexão adicional entre pontos de apoio mal localizados (CAFFARELLO, 2016). A desvantagem construtiva desse método dá-se quando existe a necessidade de elevar grandes gridshells, pois, maiores serão as forças necessárias para neutralizar o próprio peso e existe maior possibilidade da estrutura se romper (PAOLI, 2007).

Já a modelagem Descendente (downward) é um processo geralmente empregado em gridshells contemporâneos e funciona de maneira inversa à modelagem Ascendente, agindo em favor da gravidade. Dessa maneira, começa-se a construção do gridshell pelo topo e não ao nível do solo, não havendo esforços de içamento. Uma plataforma de disposição é elevada à altura desejada, na qual a malha se apoia, em um processo em que topos e vales podem ser construídos ao mesmo tempo (PAOLI, 2007). Andriaenssens (2014) classifica esse método como unstrained gridshell, ou não tensionado, pois a construção ocorre a partir da montagem de peças já curvadas, ou seja, uma estrutura rígida livre de tensões além do próprio peso.

\section{PROPRIEDADES GEOMÉTRICAS DAS MALHAS}

Segundo Williams (2014), existem alguns parâmetros no processo de form-finding de gridshells, dentre os quais destacam-se o suporte, a carga externa, a topologia do modelo e a relação da geometria com forças internas. Assim, não apenas o material garante a capacidade de suportar cargas, mas também a forma da estrutura.

Pottmann et al. (2015), afirmam que malhas híbridas, compostas por triângulos, quadrados e hexágonos, são mais otimizadas. Segundo o autor, triângulos, quadrados e hexágonos, quando agrupados, geram um fluxo de 
força mais uniforme, o que dificulta a deformação da malha.

Os triângulos, quando agrupados, geram nós compostos por seis vértices, e, portanto, malhas com boas propriedades estruturais. Contudo, resultam também em sobras de material, já que as placas são geralmente retangulares. Malhas quadradas, por sua vez, podem acarretar problemas em estruturas curvas, pois podem gerar polígonos com pequenos ângulos, além de limitar a liberdade de criação. Por sua vez, a malha hexagonal é rígida e menos suscetível à deformação, porém é desafiadora para o projeto, uma vez que cada nó possui três vértices (POTTMANN et al 2015).

Para Dimcic (2011) e Hernandes (2015), a qualidade estrutural da malha está associada à densidade da grid, que pode ser entendido como o aumento do número dos vértices em áreas estratégicas, reduzindo a área dos planos e evitando que as peças se deformem. Com essa estratégia, as forças seriam distribuídas de maneira uniforme em pontos de tensão.

Dimcic (2011) e Hernández (2015) acreditam que independente das geometrias escolhidas para compor a malha, um gridshell com qualidade estrutural é obtido por meio de processo de otimização dos padrões, que redistribui e reorienta os membros da malha, modificando a densidade da grid em uma superfície para a obtenção de uma solução ideal. Com uma otimização do padrão da grid - orientação e disposição dos perfis - pode-se obter uma minimização da curvatura dos perfis e melhorar a capacidade de carga dos gridshells (HERNÁNDEZ. p.4. 2015 - tradução nossa).

Existem diversas possibilidades de projeto para gridshell, uma vez que diferentes densidades podem ser produzidas com vários padrões. As forças internas de relaxamento e tensão podem ser controladas ajustando a densidade da malha (DIMCIC, 2011).

Em geral, esses autores concordam que a robustez do gridshell está relacionada à distribuição de carregamento ao longo de suas vigas. Quanto mais uniforme a distribuição, mais resistente é a estrutura. Por esse motivo, o padrão da malha, assim como a densidade $e$ orientação, afetam a capacidade de carga da estrutura. Esse é um estudo importante para a fase inicial de projeto, já que entre todas as geometrias possíveis, pode haver padrões ineficientes ou inviáveis (OVAL et al. 2019). Diante dessas considerações, este estudo propõe, em etapas subsequentes, a obtenção de ferramentas conceituais e práticas para aprofundar o conhecimento sobre padrões eficientes nos estágios iniciais de projeto de gridshells.

Neste estudo, são propostas duas etapas comparativas para o método: (1) Definição da modelagem por Método Construtivo Ascendente e (2) modelagem pelo Método Construtivo Descendente. No primeiro método de modelagem, a malha projetada no solo é elevada em pontos específicos, de modo que a ação da gravidade produza a forma final proposta. No segundo método, criase uma casca tridimensional, que é transformada posteriormente em uma estrutura treliçada equivalente.
A primeira etapa foi caracterizada como uma fase de préprocessamento do experimento, utilizando o plug-in Kangaroo, na qual definiu-se (a) a dimensão da estrutura a ser analisada (base e altura), (b) o número e localização dos apoios e (c) a densidade da malha. Em ambos métodos, a análise busca a menor massa, menor deslocamento dos membros e forças de utilização de compressão e tensão próximas a $100 \%$ positivo e negativo.

Em ambas as fases também foram realizados testes (d) de composição geométrica, caracterizados por treze malhas diferentes, com o objetivo de selecionar o método de modelagem, cujo desempenho estrutural seja superior em diferentes geometrias de malhas, sejam elas regulares (padronização das dimensões dos segmentos ou polígonos) ou irregulares (composições com diferentes densidades e padrões). Apesar de consolidado na literatura o uso de padrões triangulares ou diagonais para esse tipo de estrutura, testou-se casos distintos para observar as limitações e potencialidades entre métodos construtivos de modo mais abrangente.

\section{FASE DE PRÉ-PROCESSAMENTO}

$\mathrm{Na}$ fase de pré-processamento, a estrutura foi por Modelagem Ascendente utilizando a suíte de modelagem Rhinoceros3D + Grasshopper e os plug-in Kangaroo 2 e Karamba 3D. A malha foi construída em um plano XY e içada no eixo $Z$ para a posição final, produzindo uma estrutura treliçada de base quadrada, cujos lados são 25,0 metros $(25,0 \mathrm{~m} \times 25,0 \mathrm{~m})$. Por sua vez, as composições geométricas de malhas diferentes (regulares e irregulares) foram criadas com os padrões do plug-in Parakeet para Grasshopper, e três malhas regulares foram modeladas em Rhinoceros.

Tabela 1: Padrões de malha desenvolvidas pelo componente Parakeet e padrões regulares pelo Rhinoceros. Fonte: os autores.

Malhas produzidas pelo Parakeet

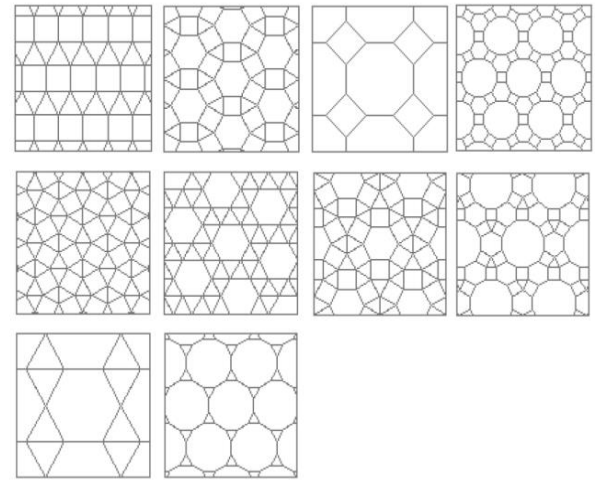

Malhas produzidas em Rhinoceros.

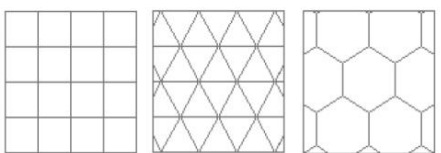


Essa estrutura foi modelada com material tubular circular com diâmetro e espessura constantes para as treze possibilidades de malhas. Apesar do Karamba 3D possuir um componente que otimiza e varia a espessura do material (cross section optimizer), para aumentar o desempenho estrutural, os testes preliminares para definição do método indicaram que este recurso aumenta significativamente a massa da estrutura.

O objetivo dessas simulações foi analisar a composição geométrica, a densidade e dimensão dos segmentos das malhas e sua relação com o desempenho estrutural. Da mesma forma, buscou-se determinar a quantidade de apoios e altura ideal da estrutura para a definição dos parâmetros adotados na próxima etapa.

As malhas geradas pelo Parakeet são compostas por módulos de geometrias diferentes, com dimensões diferentes, ou seja, não há padronização entre quantidade na subdivisão de módulos e dos segmentos dos polígonos entre todas as possibilidades malhas (Figura 1). Portanto, para avaliação e comparação das malhas foram definidas duas classificações: malhas com segmentos de dimensões padronizadas, cujas geometrias são definidas a partir de um ponto de 'origem', na extremidade do gridshell e malhas irregulares, cujo padrão geométrico inicia-se em módulo central, com recortes nas extremidades (Tabela 2 ).
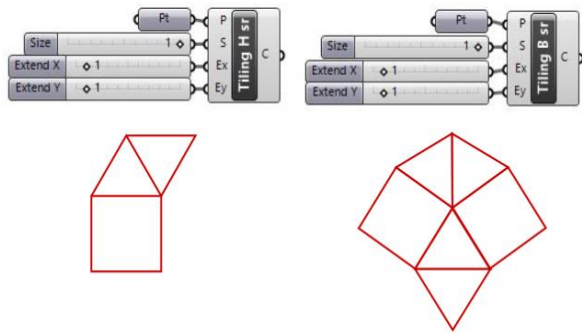

Figura 1: Padrões com composição de triângulos e quadrados, mesmo tamanho de segmentos, porém tamanho de módulos diferentes, já que a manha Tilling $\mathrm{H}$ é composta de um quadrado e dois triângulos e a malha Tilling $B$ por dois quadrados e quatro triângulos. S significa Size, ou tamanho do segmento, ajustado em $1 \mathrm{~cm}$. Ex e Ey, são números de módulos em cada eixo. $A$ imagem demonstra que para o mesmo valor associados aos eixos, os módulos apresentam tamanhos diferentes. Fonte: os autores.

Para malhas com tamanhos de segmentos padronizados, foram definidas dimensões de 1,25 metros, 2,50 metros e 5,00 metros, configurando um aumento progressivo de proporções de 1, 2 e 4 vezes o tamanho da primeira. Por sua vez, as malhas com início da composição geométrica em módulo centralizado, foram configuradas a partir da subdivisão da base quadrada em 4 módulos, 2 módulos e 1 módulo. Dessa maneira, todas as malhas foram avaliadas em ambas as categorias, em escalas e proporções semelhantes.

Tabela 2: Tabela com composição de malha em segmentos padronizados regulares e módulos centralizados, irregulares. 0 retângulo em vermelho aponta a configuração de módulos partindo do centro da malha em que os padrões são ajustados. Fonte: os autores.
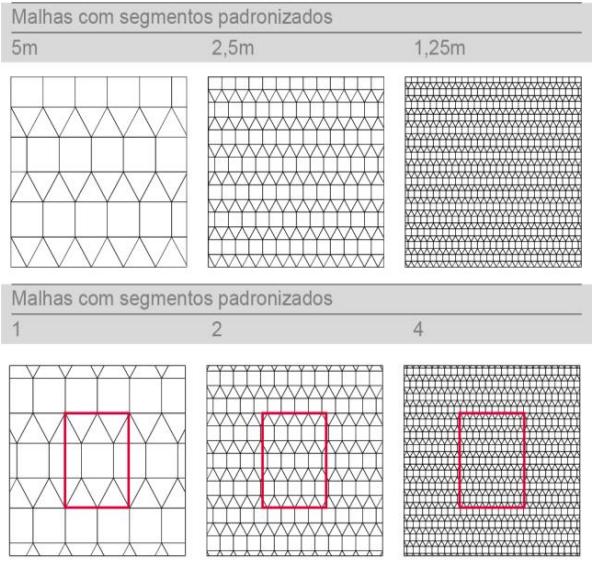

Para cada uma das malhas, foram aplicadas duas distribuições de suportes: Suportes nos quatro pontos nas extremidades (vértices), ou seis pontos (quatro nos vértices externos da base e dois no eixo central).

Para análise estrutural foram consideradas ainda as seguintes propriedades: o aço como material: Steel 235, resistente à tensões e ações externas; Dimensionamento das seções transversais das vigas: tubulares circulares com diâmetro de $13,95 \mathrm{~cm}$ e espessura entre 0,5 e 1,5 $\mathrm{cm}$; e as propriedades dos carregamentos: a gravidade e cargas imperfeitas.

\section{PRIMEIRA ETAPA: MÉTODO DE MODELAGEM ASCENDENTE}

As malhas foram obtidas utilizando os dez padrões do plug-in Parakeet para Grasshopper e três produzidos manualmente no software Rhinoceros e inseridos como curva, em substituição ao componente do Parakeet. Os pontos de suporte foram introduzidos no Grasshopper pelo componente Point. A malha, assim como os pontos de ancoragem são conectados ao componente Kangaroo Solver. O algorítmo visual possui também as definições, conforme a Figura 2.

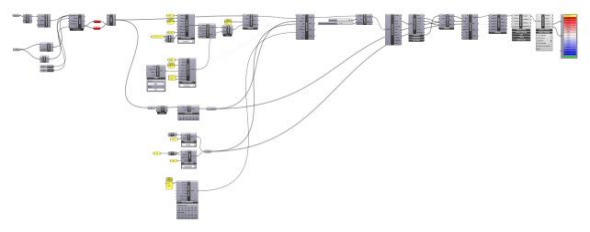

Figura 2: Algoritmo visual (GHX) desenvolvido para Modelagem Ascendente. Fonte: os autores.

Elementos de vigas: O componente explode é utilizado na malha para dividir as curvas em segmentos que serão convertidos em elementos de viga, utilizando o LineToBeam. O componente BeamJoins é utilizado para controlar as propriedades das juntas entre os nós. Para a análise, presume-se que as juntas sejam fixas.

Especificação de carregamentos: apenas o peso próprio da estrutura e forças imperfeitas foram consideradas 
nesta análise. As cargas foram projetadas em toda a superfície com valores sugeridos como padrão no Karamba3D, atuando no eixo Z negativo.

Por fim, para realizar a análise estrutural, todas as informações foram coletadas pelo componente AssembleModel, cuja primeira análise foi atribuída ao AnalizeThll. Em uma segunda análise, a saída do componente AssembleModel foi conectada ao componente LargeDeformation, com atribuição de 10,0 metros de altura à estrutura. Estes componentes estão conectados ao ModelView e o BeamView, para visualização e interpretação dos resultados.

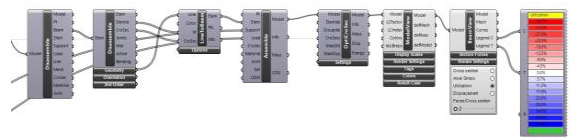

Figura 3: Componentes de Análise e visualização do Karamba3D. Fonte: os autores.

\section{SEGUNDA ETAPA: MÉTODO MODELAGEM DESCENDENTE}

$\mathrm{Na}$ segunda etapa foi concebida uma casca tridimensional em concreto, posteriormente transformada em uma estrutura treliçada equivalente. $O$ concreto foi adotado por pertencer à biblioteca do Karamba 3D, e ser um material moldável, facilmente adaptável à diferentes geometrias. Nesse caso, o processo de otimização da casca visou a aplicação da malha em uma superfície contínua, em busca de menores deformações e maior controle sobre a geometria. A estrutura também suporta vãos de 25,0 metros (definido na fase de préprocessamento da etapa de Método de Modelagem Ascendente) e é composta por perfis tubulares circulares com as mesmas características da primeira etapa.

O plug-in Octopus para Grasshopper foi utilizado na otimização, que teve como parâmetros fixos as dimensões da estrutura e as cargas aplicadas, semelhante à etapa anterior. 0 objetivo principal foi 0 melhor desempenho estrutural, com máxima altura e menor peso e deformação. Como valores resultantes da primeira etapa de pré-processamento, adotou-se a variação de altura entre 9,0 e 13,0 metros.

Além disso, a avaliação estrutural da casca pelo Karamba 3D considerou como material o concreto C30/37, o dimensionamento das seções transversais da casca com espessura entre 5,0 e 50,0 centímetros, além da gravidade como carga, uma vez que forças imperfeitas só podem ser aplicadas em elementos de vigas, assim como demonstrado na Figura 3

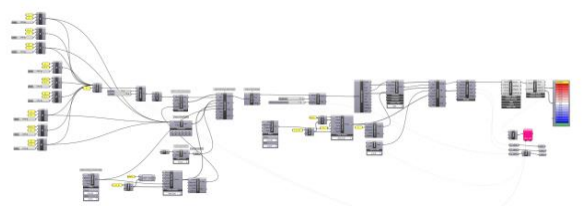

Figura 4: O algorítmo visual (GHX) desenvolvido para Casca de suporte para gridshell. Fonte: os autores.
Na elaboração da definição do modelo de casca contínua, foram definidos pontos interligados para a formação de uma superfície, transformada em mesh pelos componentes SurfaceGrid e MeshUV. Como discutido anteriormente, a densidade da triangulação da malha afeta a precisão, bem como tempo de computação do modelo (ELIASSEN; HUSEBY, 2018). A definição possui também as definições:

Elementos de casca: O componente MeshtoShell converte o mesh para elementos de shell no Karamba3D.

Especificação do material: $O$ concreto $\mathrm{C} 30 / 37$ foi selecionado entre as opções da biblioteca de materiais no Karamba3D. No componente CrossSection, a espessura da casca foi atribuída entre 5,0 e 50,0 centímetros.

Especificação dos suportes: os pontos de ancoragem são extraídos dos vértices da mesh e atribuídos ao componente Suports, semelhante ao sistema de suporte do gridshell.

Atribuição dos carregamentos: apenas o peso próprio da estrutura e forças imperfeitas foram consideradas nesta análise, como na definição da primeira etapa.

Para realizar a análise e otimização estrutural, todas as informações foram coletadas pelo componente AssembleModel, cuja primeira análise foi atribuída ao componente LargeDeformation, com variação da altura da estrutura entre 9,0 e 13,0 metros.

Então, o modelo foi submetido a uma segunda análise pelo componente OptimizeCrossSection, capaz de escolher entre os valores da variação da espessura da malha em pontos específicos, atribuída ao CrossSection. Do componente OptimizeCrossSection existem saídas com valores de deformação e massa que são atribuídos ao motor de otimização Octopus. Estes componentes estão conectados ao ModelView e o ShellView, para visualização e interpretação dos resultados.

Para estudar o comportamento estrutural o Gridshell resultante da casca, foram considerados parâmetros semelhantes à etapa de Método de Modelagem Ascendente: O aço (Steel 235) como material; no componente CrossSection, foi selecionada a forma tubular circular com diâmetro e espessura usados para toda a análise (diâmetro de $13,95 \mathrm{~cm}$ e espessura entre 0,5 e 1,5 centímetros), demostrado na Figura 4.

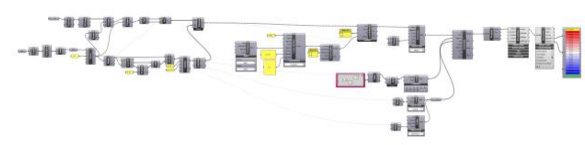

Figura 5: Script desenvolvido para projeção da malha sobre o Mesh. Fonte: os autores.

Para a configuração da malha, a estrutura resultante da otimização é inserida em um novo arquivo do Rhinoceros e no Grasshopper como componente mesh, enquanto os padrões de malha são inseridos como curvas e projetados na superfície do mesh. O componente explode é aplicado para dividir segmentos de curvas e transformados em vigas pelo componente LineToBeam. 
O material, sua seção transversal, carregamentos, análise e visualização são configurados como o script da primeira etapa.

\section{RESULTADOS}

DESEMPENHO DA PRIMEIRA FASE DE SIMULAÇÃO: MÉTODO DE MODELAGEM ASCENDENTE

A primeira simulação por meio do Karamba3D foi realizada para gridshells com treze composições de malhas utilizando o método da catenária. Analisou-se o comportamento das treze estruturas, com variação de número de apoios (quatro e seis, conforme apresentado na metodologia). Do ponto de vista de desempenho estrutural, ambas apresentam comportamento semelhante, com pequena variação entre os valores de compressão, tensão e massa. No entanto, optou-se pela estrutura de quatro apoios, nos vértices das extremidades, já que são capazes de vencer o mesmo vão, sem apoios intermediários, com maior ganho de altura.

Tabela 3: Análise de comparação entre estruturas com quatro e seis apoios em malha composta por quadrados e triângulos. Legenda: U(C)- Utilização de Compressão; U(T)- Utilização de Tensão; M- massa; Altura que a estrutura atingiu e Imagem da estrutura. Fonte: os autores.

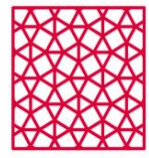

\begin{tabular}{llllll}
\hline Apoios $U(\mathrm{C})$ & $\mathbf{U}(\mathrm{T})$ & $\mathrm{M}(\mathrm{kg})$ & Altura $(\mathrm{m})$ & Imagem \\
\hline 4 & $-3.2 \%$ & $11.1 \%$ & 23071.8 & 9.5 \\
\hline 6 & $-2.7 \%$ & $6.8 \%$ & 22766.1 & 6.2 \\
\hline
\end{tabular}

Após a determinação dos apoios, foi realizada a comparação entre a composição de malhas com segmentos de dimensões iguais e a subdivisão da malha em módulos. Malhas com tamanhos de segmentos padronizados, com dimensões de 5,00 metros, 2,50 metros e 1,25 metros foram comparadas às malhas com padrões geométricos arranjados em modulação central com quatro, dois e um módulo.

Tabela 4: Comparação entre malhas de segmentos padronizados e módulos centralizados com mesma composição geométrica de quadrados e triângulos. Legenda: SP - malha com segmentos padronizados; MC - malha com módulo centralizado; U(C)- Utilização de Compressão; U(T)- Utilização de Tensão; M- massa; Altura que a estrutura atingiu e Imagem da estrutura. Fonte: os autores.

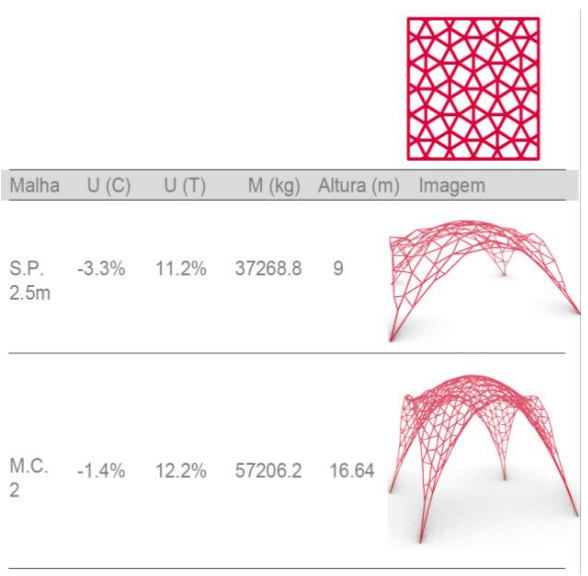

Notou-se que estruturas com padrões geométricos centralizados em direção às bordas, aumentavam significativamente a massa, mas não a capacidade de suporte das estruturas. Assim, optou-se por malhas com segmentos de dimensões iguais, que começam na origem da base da estrutura, como mais eficientes, já que facilitam a fabricação com a produção seriada das barras (Tabela 02). Além disso, segmentos com 2,50 metros, ou seja, 1/10 do vão da estrutura, produziram gridshells com maior capacidade de carga e menor massa em comparações às demais proporções.

Os segmentos das malhas centralizadas, no entanto, como não tiveram as dimensões pré-fixadas, sofreram grande deformação, como consequência da atuação do plug-in Kangaroo na construção do script. Além disso, essas também geram barras de dimensões desiguais, uma vez que elas sofrem recortes nas extremidades da estrutura. O experimento também demonstrou que malhas que atingiam entre 9,00 e 13,00 metros de altura alcançavam curvaturas com melhor desempenho estrutural. Portanto, esse intervalo de valores tornou-se parâmetro para a otimização da próxima fase.

\section{DESEMPENHO DA SEGUNDA FASE DE SIMULAÇÃO: MÉTODO DE MODELAGEM DESCENDENTE}

Na segunda etapa de simulação, foi produzida uma casca de concreto que simularia o suporte para a construção de um gridshell com forma correspondente. A otimização foi realizada usando o Octopus, um plug-in para Grasshopper que desenvolve otimização baseada em simulação usando algoritmos genéticos. Esse plug-in foi desenvolvido com base em princípios evolucionários multiobjetivo, ou seja, possui a capacidade de fazer referência cruzada de vários parâmetros simultaneamente. Desse modo, produz diversas soluções otimizadas entre os extremos de cada meta (SANTANA, 2015).

A casca otimizada teve como objetivo melhorar sua resistência à carga, porém a partir de pequenos ajustes na forma, resultante da primeira etapa de simulação. A otimização foi configurada para minimizar a massa e o deslocamento, atingindo máxima altura. No entanto, segundo Dźwierzyńska (2020), a minimização de massa e deflexão são objetivos conflitantes, ou seja, quanto 
maior a massa, menor deslocamento, enquanto menor é a massa, maior também é o deslocamento.

Em simulação, no gráfico da Fronte de Pareto, os indivíduos mais próximos da curva são os casos ideais para os três objetivos. Para a essa pesquisa, foi escolhido o indivíduo mais próximo da origem com maior altura, demonstrado na Tabela 5.

Tabela 5: Casca resultante do processo de otimização. Legenda: U(C)- Utilização de Compressão; U(T)- Utilização de Tensão; Mmassa; Altura que a estrutura atingiu e Imagem da estrutura. Fonte: os autores.

\begin{tabular}{ccccc}
\hline$U(\mathrm{C})$ & $U(\mathrm{~T})$ & $\mathrm{M}(\mathrm{kg})$ & Altura $(\mathrm{m})$ & Imagem \\
\hline$-95.5 \%$ & $55.7 \%$ & 83998.8 & 10
\end{tabular}

Em seguida, o modelo otimizado foi convertido em um gridshell correspondente, aplicando as malhas com segmentos de mesma dimensão, com 5,0 metros, 2,50 metros e 1,25 metros para as treze composições de malha. De modo análogo, as malhas com segmentos padronizados da primeira etapa (Método de Modelagem Ascendente) foram submetidas a uma nova simulação, desta vez atribuindo uma altura de 10 metros, para facilitar a comparação entre ambos os métodos.

Tabela 6: Comparação entre métodos de modelagem em malha triangular com segmentos padronizados. Legenda: MC- Método Construtivo; M. A. - Método de Modelagem Ascendente; M.D.Método de Modelagem Descendente; U(C)- Utilização de Compressão; U(T)- Utilização de Tensão; M- massa; Altura que a estrutura atingiu e Imagem da estrutura. Fonte: os autores.

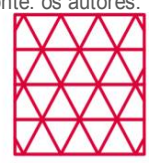

\begin{tabular}{lcccc}
\hline M.C. & $\mathrm{U}(\mathrm{C})$ & $\mathrm{U}(\mathrm{T})$ & $\mathrm{M}(\mathrm{kg})$ & Altura $(\mathrm{m})$ \\
\hline M.A. & $-47.5 \%$ & $66.6 \%$ & 17008.2 & 9.5 \\
\hline & & & & \\
M.D. & $-72.1 \%$ & $71.3 \%$ & 43863.2 & 10
\end{tabular}

Notou-se que, apesar do Método de Modelagem Ascendente ser o mais recorrente em estudos sobre tema, o Método de Modelagem Descendente gerou estruturas com melhor desempenho estrutural. Para as mesmas composições de malha, a massa da estrutura manteve-se a mesma em ambos os métodos, porém, observou-se melhor desempenho do Método Descendente comparando-se os dados de utilização de tensão e compressão. Além disso, essa melhora significativa demonstra que o gridshell otimizado trouxe ganhos em curvatura e altura, independente da composição da malha. No Método Descendente, o plug-in Kangaroo não foi utilizado e, portanto, não houve deformação das peças, que se mantiveram da mesma dimensão para facilitar a fabricação.

Em relação à composição geométrica, como esperado, percebeu-se que malhas triangulares geraram estruturas mais rígidas, em decorrência dos nós com seis vértices. Entretanto, notou-se comportamentos semelhantes com malhas que combinam a composição de quadrados e triângulos ou malhas compostas por triângulos e hexágonos, sugerindo que o arranjo em diagonal seja o elemento estruturador.

Tabela 7: Comparação entre padrões diferentes padrões com Método de Modelagem Descendente. Legenda: MC- Método de Modelagem Descendente; U(C)- Utilização de Compressão; U(T)- Utilização de Tensão; M- massa; Altura que a estrutura atingiu e Imagem da estrutura. Fonte: os autores.

\begin{tabular}{|c|c|c|c|c|}
\hline M.D. & $U(\mathrm{C})$ & $U(T)$ & $M(\mathrm{~kg})$ & Altura $(\mathrm{m})$ Imagem \\
\hline $\mathrm{m}$ & $-72.2 \%$ & $66.6 \%$ & 35466.3 & 10 \\
\hline$A x$ & $-72.1 \%$ & $71.3 \%$ & 43863.2 & 10 \\
\hline 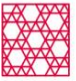 & $-77.5 \%$ & $76.6 \%$ & 32731.6 & 10 \\
\hline
\end{tabular}

Dźwierzyńska (2018) em sua pesquisa descreve que malhas com parâmetro de "utilização" que apresentam resultados muito discrepantes entre compressão e torção, deveriam ser adensadas nos pontos de tensão ou incrementadas no número de suporte. Para a nossa pesquisa, como os números de suporte são constantes, os resultados de utilização serão os indicadores de alterações e adensamento de composição das malhas.

Tabela 8: Comparação entre valores de Utilização em malhas compostas por quadrados e triângulos. Legenda: MD- Método de Modelagem Descendente; U(C)- Utilização de Compressão; U(T)- Utilização de Tensão; M- massa; Altura que a estrutura atingiu e Imagem da estrutura. Fonte: os autores

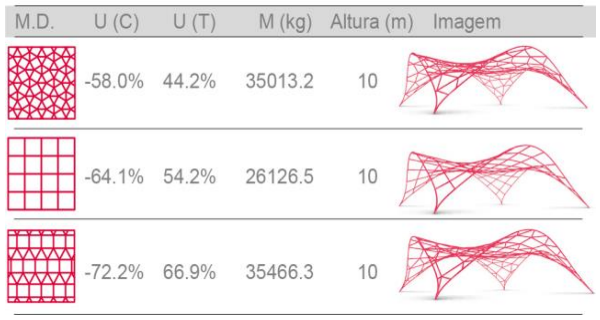

Malhas compostas por quadrados ou quadrados e triângulos na diagonal, demonstram possibilidade de adensamento próxima aos suportes para garantir melhor desempenho estrutural. Isso significaria um incremento da massa da estrutura, porém também menores deformações. Essas informações são essenciais para a continuidade da pesquisa, e serão aprofundadas na etapa de análises de composição geométrica, como estágio preliminar à elaboração de Gramática da Forma.

\section{CONCLUSÃO}

Os algorítmos visuais elaborados para os Métodos de Modelagem Ascendente e Descendente podem ser utilizados para qualquer forma de gridshells, com diversos padrões de composição internos de malhas, além de permitir a introdução manual de geometrias por segmentos ou por modelos matemáticos inseridos no Grasshopper. 
A intenção deste trabalho foi demonstrar uma abordagem de modelagem paramétrica com testes de desempenho estrutural de um gridshell em seu estágio inicial de projeto, considerando o método de modelagem e as características geométricas da estrutura. Apesar de estruturas com Modelagem Ascendente serem mais comuns na literatura, percebeu-se que o método Descendente gerou estruturas com melhor desempenho estrutural.

A partir do método estabelecido e testes compositivos, foram realizados testes de modificação de parâmetros que impactam diretamente o desempenho estrutural, tais como a dimensão dos segmentos das peças, a altura da estrutura, número de suportes, composição e densidade da malha. Esse experimento atua como base para um estudo futuro que visa a produção de malhas otimizadas com uso da gramática da forma como processo generativo de projeto. Informações sobre densidade, direção das peças, número de nós, dentre outras, serão abordadas em trabalhos futuros como estratégias para a criação ou aplicação das regras, com o objetivo de incremento do desempenho estrutural a partir da geometria das malhas.

\section{AGRADECIMENTOS}

Agradecemos ao Nó.Lab (DAU/UFV) pelo suporte para o desenvolvimento desta pesquisa ao CAPES, Coordenação de Aperfeiçoamento de Pessoal de Nível Superior, pela bolsa de Mestrado vinculada a este projeto. Também agradecemos ao Programa de PósGraduação em Arquitetura e Urbanismo da Universidade Federal de Viçosa (UFV) pelo auxílio financeiro para apresentação desse trabalho no SIGraDi.

\section{REFERÊNCIAS}

Adriaenssens, S., Block, P., Veenendaal, D., \& Williams, C. (2014). Shell structures for architecture: Form finding and optimization. In Shell Structures for Architecture: Form Finding and Optimization (1st ed., Vol. 9781315849). Routledge. https://doi.org/10.4324/9781315849270.

Carvalho, D.F.P.A. (2015). Gidshells em madeira: morfologia, aplicabilidade, comportamento estrutural e projeto. Dissertação de Mestrado, Universidade de Coimba, Coimbra, Portugal.

Chilton, J. (2016). Timber gridshells: Architecture, structure and craft (10 ed). London, U. K: Routledge.

Dimcic. M (2011). Structural Optimization of Grid Shells based on Genetic Algorithms Stuttgart. Dissertação de Doutorado. Universit"at Stuttgart, Alemanha.

Dzwierzynska, J. (2018). Shaping curved steel rod structures. Czasopismo https://doi.org/10.4467/2353737XCT.18.117.8892

Dzwierzynska, J. (2020). Multi-objective optimizing curvilinear steel bar structures of hyperbolic paraboloid canopy roofs. Buildings, 10(3),39.

https://doi.org/10.3390/buildings 10030039
Eliassen, M. Huseby, A.(2018) The Digital Workflow of Parametric Structural Design Developing Grid Shells in a Nordic Climate. Tese de Mestrado, University in Trondheim, Noruega.

Hernández, C. H. (2015). Recent developments in architectural fabric structures in Latin America. In Fabric Structures in Architecture (p.585-660).Elsevier. https://doi.org/10.1016/B978-1-78242-233-4.00017-6

Kunz, M., \& Prauchner, M. B. (2015). Uso do sistema estrutural gridshell na criação de formas complexas em estruturas de madeira. Revista de Arquitetura IMED, 4(1), 19-25. https://doi.org/10.18256/2318-1109/arqimed.v4n1p19-25

Love, A. E. H. (1888). The Small Free Vibrations and Deformation of a Thin Elastic Shell. Philosophical Transactions of the Royal Society A: Mathematical, Physical and Engineering Sciences, 179(0), 491546. doi:10.1098/rsta.1888.0016

Naicu, D. I. (2012). Geometry and performance of timber gridshells. Dissertação de mestrado. University of Bath, Baht, Inglaterra.

Oval, R., Rippmann, M., Mesnil, R., Van Mele, T., Baverel, O., \& Block, P. (2019). Feature-based topology finding of patterns for shell structures. Automation in Construction, 103, 185201. https://doi.org/10.1016/j.autcon.2019.02.008

Paoli, C. (2007). Past and future of grid shell structures. Dissertação de Mestrado, Massachusetts Institute of Technology - MIT, Massachusetts, Estados Unidos da América.

Pone, S. D'amico, B. Colabella, S. Flore. A. (2013). Timber post formed gridshell: digital forma finding/ drawing and building tool. In: Proceedings of the International Association for Shell and Spatial Structures (IASS) Symposium. Retrieved from: https://www.researchgate.net/publication/261285620 Timber _post_formed_grid_shell_digital_form_finding_drawing_and building tool.

Pottmann, H., Eigensatz, M., Vaxman, A., \& Wallner, J. (2015). Architectural geometry. Computers \& Graphics, 47, 145-164 https://doi.org/10.1016/j.cag.2014.11.002

Preisinger, C. (2016). Parametric structural modeling Karamba: User Manual For version 1.2.2. Retrieved from http://web.arch.virginia. edu/struct/ arch721/docs/Karamba_1_2_2_Manual.pdf.

Rieffel, J, Valero-Cuevas, F., Lipson, H., (2009) "Automated discovery and optimization of large irregular tensegrity structures",Computers and Structures, Vol. 87, pp. 368-379.

Santana, L.O; Guimarães, İ.B.B; Carlo, J.C., 2015 Parametrização aplicada ao desempenho energético de edificações. V!RUS, São Carlos, 11.[online] Retrieved from: http://www.nomads.usp.br/virus/virus $11 /$ ?sec=4\&item=4\&lang $=p t$

Toussaint, M. H.Ba.(2007). Design Tool for Timber Gridshells. Dissertação de mestrado. Delft University of Technolog, Delft, Holanda.

Williams, C. (2014). What is a shell? In Adriaenssens, S., Block, P., Veenendaal, D., \& Williams, C. (Eds.). Shell Structures for Architecture: Form Finding and Optimization (pp 21-31). London, U. K: Routledge. 